\title{
Utility of Survivin, BAP1, and Ki-67 immunohistochemistry in distinguishing epithelioid mesothelioma from reactive mesothelial hyperplasia
}

\author{
KEI KUSHITANI ${ }^{1}$, VISHWA JEET AMATYA ${ }^{1}$, AMANY SAYED MAWAS ${ }^{2}$, RUI SUZUKI ${ }^{1}$, YOSHIHIRO MIYATA ${ }^{3}$, \\ MORIHITO OKADA $^{3}$, KOUKI INAI ${ }^{1}$, TAKUMI KISHIMOTO ${ }^{4}$ and YUKIO TAKESHIMA ${ }^{1}$ \\ ${ }^{1}$ Department of Pathology, Institute of Biomedical and Health Sciences, Hiroshima University, \\ Hiroshima 734-8551, Japan; ${ }^{2}$ Department of Pathology and Clinical Pathology, Faculty of Veterinary Medicine, \\ South Valley University, Qena 83523, Egypt; ${ }^{3}$ Department of Surgical Oncology, Research Center for \\ Radiation Casualty Medicine, Research Institute for Radiation Biology and Medicine, Hiroshima University, \\ Hiroshima 734-8551; ${ }^{4}$ Department of Internal Medicine, Okayama Rosai Hospital, Okayama 702-8055, Japan
}

Received June 30, 2017; Accepted November 20, 2017

DOI: $10.3892 / \mathrm{ol} .2018 .7765$

\begin{abstract}
Histological distinction between epithelioid mesothelioma (EM) and reactive mesothelial hyperplasia $(\mathrm{RMH})$ can be challenging. The aim of this study was to assess the diagnostic utility of Survivin, Ki-67, and loss of BRCA1-associated protein 1 (BAP1) expressions in distinguishing EM from RMH using immunohistochemistry. Formalin-fixed, paraffin-embedded specimens from 78 cases of EM and 80 cases of RMH were immunohistochemically examined for Survivin, BAP1, and Ki-67. In addition, receiver operating characteristic curve analyses were performed to establish the cut-off values for Survivin and Ki-67 labelling indices. Survivin (cut-off value: $5 \%$ ) had $67.7 \%$ sensitivity and $100 \%$ specificity, while Ki-67 (cut-off value: 10\%) had $85.1 \%$ sensitivity and $87.5 \%$ specificity, and BAP1 had $66.2 \%$ sensitivity and $100 \%$ specificity for the differentiation of EM from RMH. Among the combinations of two markers, the combination of Survivin and BAP1 (Survivin-positive and/or BAP1-loss finding) had the highest diagnostic accuracy (sensitivity: $89.8 \%$; specificity: 100\%; accuracy: 95.3\%). We recommend using the combination of Survivin and BAP1 to distinguish EM from RMH.
\end{abstract}

\section{Introduction}

Malignant mesothelioma (MM) is a relatively rare but highly aggressive malignant neoplasm arising from mesothelial cells of the pleura, peritoneum, pericardium, and tunica vaginalis.

Correspondence to: Professor Yukio Takeshima, Department of Pathology, Institute of Biomedical and Health Sciences, Hiroshima University, 1-2-3 Kasumi, Minami-ku, Hiroshima 734-8551, Japan E-mail: ykotake@hiroshima-u.ac.jp

Key words: BAP1, immunohistochemistry, Ki-67, mesothelioma, reactive mesothelial hyperplasia, survivin
It is well-correlated with occupational and environmental asbestos exposure. $(1,2)$ The incidence of MM has increased in many countries; (3) in Japan, mortality due to MM has increased since the 1990s, and is predicted to peak in the 2030s (4).

Epithelioid mesothelioma (EM) must be differentiated from reactive mesothelial hyperplasia $(\mathrm{RMH})$, which is a non-neoplastic condition frequently caused by pleuritis, peritonitis, or serosal invasion of other cancers. Due to the close resemblance of EM to $\mathrm{RMH}$, differentiation by routine histological observation alone can be challenging.

Various established and novel immunohistochemical markers have been utilized to distinguish EM from other malignancies (5-8) and RMH (6,9-17) Multiple potential immunohistochemical markers, including Ki-67, desmin, epithelial membrane antigen (EMA), p53, glucose transporter 1, insulin-like growth factor 2 messenger RNA binding protein-3 and BRCA1-associated protein 1 (BAP1) have been evaluated. However, despite the use of these immunohistochemical markers, the distinction between EM and RMH remains challenging in some cases.

Recently, detection of p16 (CDKN2A) homozygous deletion (p16 HD) using fluorescence in situ hybridization (FISH) has been used to differentiate MM from RMH, with $100 \%$ specificity. However, the sensitivity of this marker for pleural EM varies between 45 and $86 \%$, while its sensitivity for peritoneal EM ranges from 14 to $41 \%$ in different laboratories (10,18-20). In our unpublished experience, p16 HD (detected by FISH) was present in $63.2 \%(12 / 19)$ of EM cases, but absent in all RMH cases $(0 / 20)$. Although the detection of p16 HD using FISH may be considered highly specific, its sensitivity in differentiating EM from RMH is not very high. In addition, FISH analysis cannot be applied in all cases or in all pathology laboratories, given its high cost and stringent experimental requirements.

We recently reported that phorbol 12-myristate-13-acetate-induced protein-1 (PMAIP-1; Noxa) and baculoviral IAP repeat-containing 5 (BIRC5; Survivin) mRNA expression levels are significantly higher in EM than in non-neoplastic pleural tissue, and discussed the utility of anti-Noxa antibody 
for the distinction between EM and RMH (21). However, the utility of Survivin IHC for the differentiation of benign and malignant mesothelial proliferation has not yet been assessed.

Here, we studied the utility of Survivin and Ki-67 expressions along with the loss of BAP1 expression in distinguishing benign from malignant mesothelial proliferation.

\section{Materials and methods}

Patients and histological samples. We used formalin-fixed, paraffin-embedded (FFPE) specimens from 78 patients with a definite histological diagnosis of EM who had undergone thoracoscopic pleural biopsy, pleurectomy/decortication, extrapleural pneumonectomy, or autopsy between 2000 and 2016 . FFPE histological samples from surgical specimens obtained from 80 patients with a histological diagnosis of RMH were obtained via thoracoscopic biopsy, laparoscopic biopsy, or surgical resection between 2005 and 2016. These samples were retrieved from the archives of the Department of Pathology at Hiroshima University (Hiroshima, Japan). Each of the tumour specimens was independently reviewed by three pathologists (K.K., V.J.A, and Y.T.), and all cases of mesothelioma were diagnosed according to currently accepted World Health Organization Histological Criteria $(6,22)$.

The tissue samples were retrieved from the archive of the Department of Pathology at Hiroshima University's Institute of Biomedical and Health Sciences. The collection of tissue specimens for this study was carried out in accordance with the 'Ethics Guidelines for Human Genome/Gene Research' enacted by the Japanese Government. Ethical approval was obtained from the institutional ethics review committee (Hiroshima University E-974). All experimental procedures were in accordance with the with ethical guidelines.

Immunohistochemical procedures. Immunohistochemical staining of sections from the FFPE tissue samples was performed using Ventana BenchMark GX (Roche Diagnostics, Basel, Switzerland). In brief, after deparaffinization using EZ-Prep (Roche Diagnostics) and antigen retrieval using Cell Conditioning 1 buffer at $95^{\circ} \mathrm{C}$ for $32 \mathrm{~min}$, sections were incubated with primary antibodies. The primary antibodies were anti-Survivin (cat. no. AF886, polyclonal, dilution of 1:200; R\&D systems, Minneapolis, MN, USA), anti-BAP1 (C-4, dilution of 1:50; Santa Cruz Biotechnology, Inc., Dallas, TX, USA), and anti-Ki-67 (MIB-1, dilution of 1:25; Dako, Glostrup, Denmark). Incubation with secondary antibodies and detection was performed using the Ventana UltraView Universal DAB Detection kit.

Nuclear staining of Survivin, BAP1, and Ki-67 in EM or $\mathrm{RMH}$ cells with the same or higher intensity than internal positive controls was regarded as positive staining. Negative staining of BAP1 was defined as completely absent nuclear staining in the target cells in the presence of a positive internal control such as lymphocytes or stromal cells. Although some cases had weak cytoplasmic positivity for Survivin and BAP1, we have not included cases with only cytoplasmic positivity for Survivin and BAP1 for evaluation in this study. Immunoreactivity of Survivin and Ki-67 was evaluated using a labelling index (\% of positive cells) in the 'hot spot' exhibiting the highest number of positive cells compared to the rest of the lesion. We evaluated at least 100 (maximum 500) EM or RMH cells in high power fields (x400). Counting of labelling indices of Survivin and Ki-67 was performed by three pathologists (K.K., V.J.A, and Y.T.) independently; the mean of three numbers was then calculated.

Statistical analysis. Receiver operating characteristic (ROC) curve analysis was performed to establish the cut-off values for the Survivin and Ki-67 labelling indices. The cut-off points were determined based on the Youden index. All statistical analyses were performed using EZR (Saitama Medical Center, Jichi Medical University, Saitama, Japan), a graphical user interface for R (The R Foundation for Statistical Computing, Vienna, Austria). More precisely, it is a modified version of $\mathrm{R}$ commander designed to add statistical functions frequently used in biostatistics (23).

Sensitivity, specificity, positive predictive values, negative predictive values, and diagnostic accuracies were calculated for each marker and combinations of two markers.

\section{Results}

Survivin expression and cut-off value. Representative immunohistochemical staining images for EM and RMH are shown in Fig. 1. Survivin expression was significantly higher in EM than in RMH. The mean of the Survivin labelling indices in EM [mean, 9.3; range, 0-24.5, standard deviation (SD), 6.5] was significantly higher than that in RMH (mean, 1.2; range, 0-4.0, $\mathrm{SD}, 1.2)$ (t-test, P-value <0.001). Distributions of the Survivin labelling indices in EM and RMH are shown in Fig. 2A.

The cut-off value for the Survivin IHC assay led by the result of ROC analysis was 4.000 (Fig. 2B). Based on the ROC analysis, and in consideration of convenience in practical pathological diagnosis, we set the cut-off value for the Survivin IHC assay at 5\%. Immunoreactivity of Survivin was classified as negative (positivity of less than $5 \%$ of the mesothelioma cells or non-neoplastic mesothelial cells) or positive (positivity of over $5 \%$ of the mesothelioma or mesothelial cells).

Forty-two of $62(67.7 \%)$ EM cases were positive for Survivin. In contrast, none of the RMH cases were positive for Survivin (Table I).

Ki-67 expression and cut-off value. Representative immunohistochemical staining images for EM and RMH are shown in Fig. 3. Ki-67 expression was also significantly higher in EM than in RMH. The mean of the Ki-67 labelling indices in EM (mean, 32.6; range, 1.0-90.0; SD, 22.1) was significantly higher than that in $\mathrm{RMH}$ (mean, 3.5; range, 0-20.0, SD, 4.2) (t-test, $\mathrm{P}$-value $<0.001)$. Distributions of the Ki-67 labelling indices in EM and RMH are shown in Fig. 4A.

The cut-off value for the Ki-67 IHC assay led by the result of ROC analysis was 10.333 (Fig. 4B). Based on the ROC analysis, and in consideration of convenience in practical pathological diagnosis, we set the cut-off value for the Ki-67 IHC assay at $10 \%$. Immunoreactivity of $\mathrm{Ki}-67$ was classified as negative (positivity of less than $10 \%$ of the mesothelioma cells or non-neoplastic mesothelial cells) or positive (positivity of over $10 \%$ of the mesothelioma or mesothelial cells).

Fifty-seven of 67 (85.1\%) EM cases and 7 of 56 (12.5\%) RMH cases were positive for Ki-67 (Table I). 
A

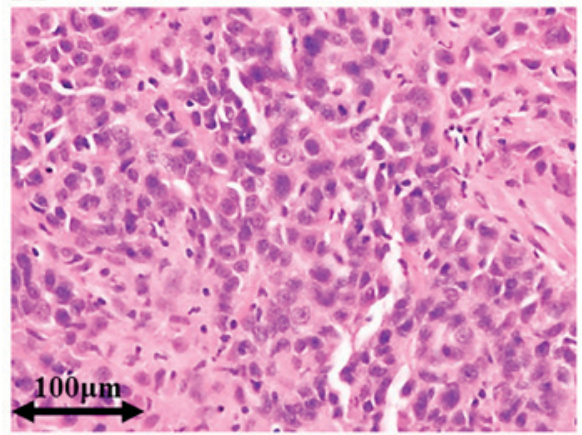

C

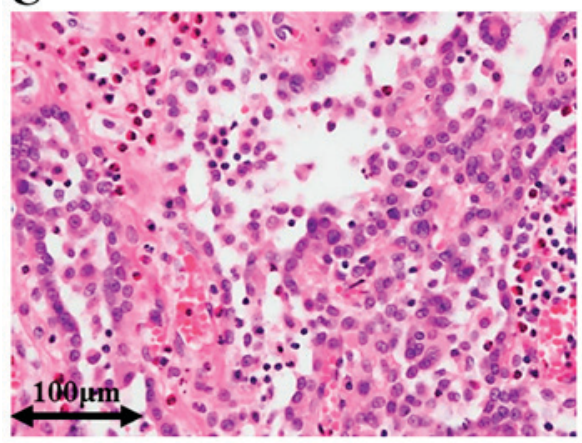

B

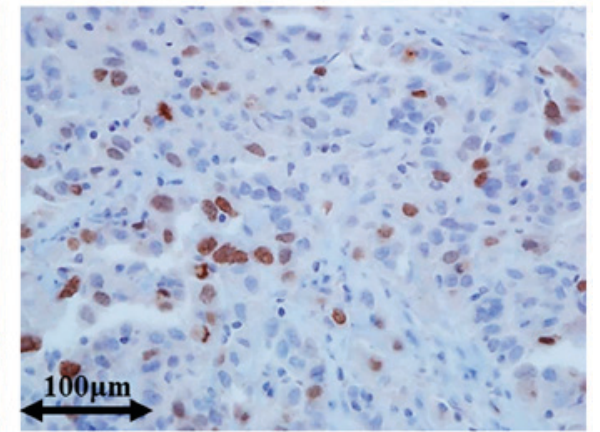

D

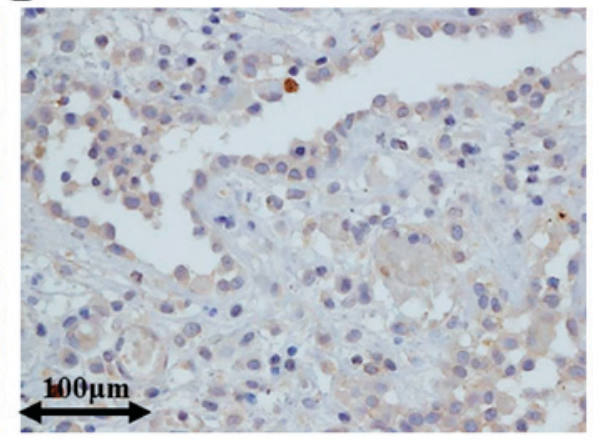

Figure 1. Representative histological images of Survivin IHC. (A) EM with H\&E staining. (B) Survivin IHC in EM; labelling index, 18.1. (C) RMH with H\&E stain. (D) Survivin IHC in RMH; labelling index, 1.3. IHC, immunohistochemistry; EM, epithelioid mesothelioma; RMH, reactive mesothelial hyperplasia; $\mathrm{H} \& \mathrm{E}$, haematoxylin and eosin.

A

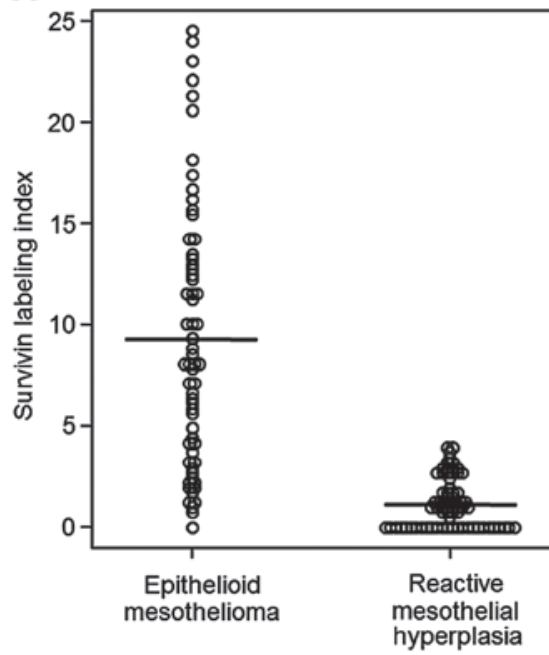

B

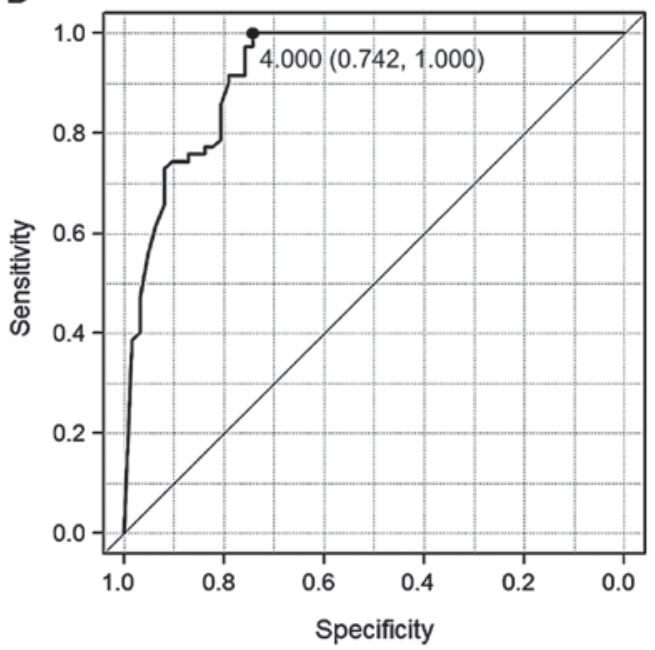

Figure 2. (A) Distribution of Survivin labelling index in epithelioid mesothelioma and reactive mesothelial hyperplasia. The horizontal line in the dot chart shows the mean. (B) ROC analysis. ROC curve was estimated using Survivin labelling index. Cut-off value based on the Youden index is also shown. ROC, receiver operating characteristic.

BAP1 expression. Loss of nuclear BAP1 expression was observed in 49 of 74 (66.2\%) cases of EM (Table I). Almost all cases without BAP1 expression had a homogenous expression loss pattern. No heterogeneous loss patterns were observed. In contrast, nuclear BAP1 expression was preserved in all 78 RMH cases (Table I). Representative immunohistochemical staining images for EM and RMH are shown in Fig. 5.

Utilities of each marker and combinations of two markers. The sensitivity and specificity of each marker and combinations of two markers for the distinction between EM and RMH are shown in Table II. Among three single markers and six combination patterns of two markers, 'Survivin-positive and/or BAP1-loss' finding showed the highest diagnostic accuracy (95.3\%).

\section{Discussion}

Accurate histopathological differentiation between MM and RMH is extremely important, not only for clinical management, but also for the appropriate operation of the public 
Table I. Immunohistochemical findings of Survivin, Ki-67, and BAP1 in epithelioid mesothelioma and reactive mesothelial hyperplasia.

\begin{tabular}{|c|c|c|c|c|c|c|}
\hline \multirow{2}{*}{$\begin{array}{l}\text { Immunohistochemical } \\
\text { data }\end{array}$} & \multicolumn{3}{|c|}{ Epithelioid mesothelioma } & \multicolumn{3}{|c|}{ Reactive mesothelial hyperplasia } \\
\hline & $\mathrm{n}(\%)$ & Negative & Positive & $\mathrm{n}(\%)$ & Negative & Positive \\
\hline Survivin expression & $42 / 62(67.7)$ & 20 & 42 & $0 / 70$ & 70 & 0 \\
\hline Ki-67 expression & $57 / 67(85.1)$ & 10 & 57 & $7 / 56(12.5)$ & 49 & 7 \\
\hline BAP1-loss & $49 / 74(66.2)$ & 25 & 49 & $0 / 78$ & 78 & 0 \\
\hline
\end{tabular}

BAP1, BRCA1-associated protein 1 .
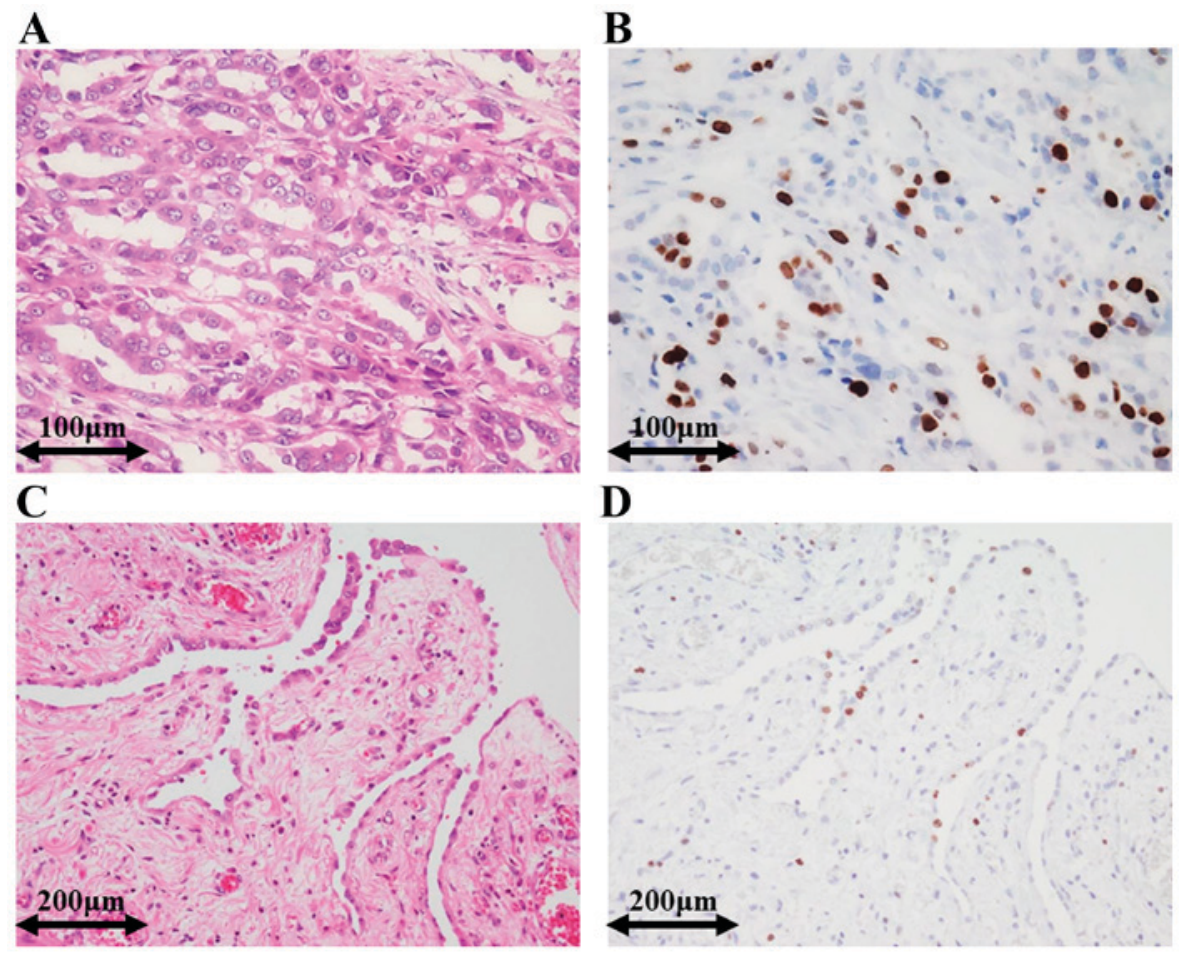

D

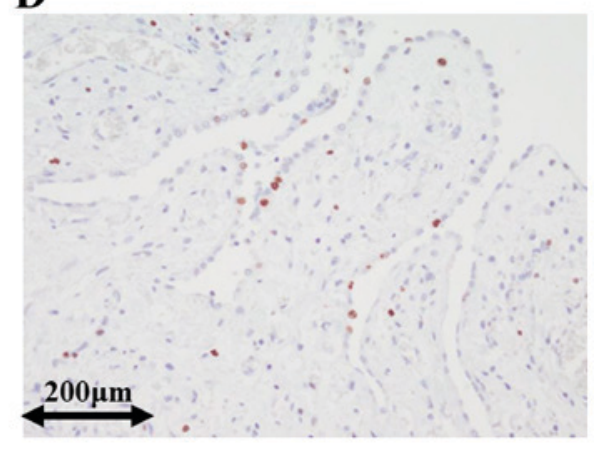

Figure 3. Representative histological images of Ki-67 IHC. (A) EM with H\&E stain. (B) Ki-67 IHC in EM; labelling index, 35.0. (C) RMH with H\&E stain. (D) Ki-67 IHC in RMH; labelling index, 8.7. IHC, immunohistochemistry; EM, epithelioid mesothelioma; RMH, reactive mesothelial hyperplasia; H\&E, haematoxylin and eosin.

compensation system for victims of environmental and occupational asbestos exposure and their dependents. To obtain a better marker for EM, we evaluated the diagnostic utilities of Survivin, BAP1, and Ki-67 in differentiating EM from RMH. We found that the sensitivity and specificity of the nuclear Survivin labelling index following the use of a properly determined cut-off value was appropriate in distinguishing EM from RMH. The utility of Survivin IHC for the differentiation between benign and malignant mesothelial proliferation has not been reported to date. To the best of our knowledge, this is the first report evaluating the utility of Survivin IHC in differentiating EM from RMH.

Survivin is the smallest member of the inhibitor of apoptosis (IAP) family, and is expressed highly in most human foetal tissues and cancers. However, it is completely absent in terminally-differentiated tissues. Survivin functions as a regulator of both cell division and apoptosis. The function of
Survivin differs according to cellular localization. Cytosolic Survivin is believed to function as an apoptotic suppressor, while nuclear Survivin is postulated to regulate cell division (24). Overexpression of Survivin is associated with tumour progression and poor prognosis in many types of human malignancies, including MM $(25,26)$. In fact, several reports indicate that Survivin is a promising marker for the diagnosis of malignant pleural effusion (27). Survivin has also been reported to be associated with anti-tumour activity and outcomes of chemotherapy in MM, and is a new therapeutic target for the treatment of MM (28-30).

While the Survivin labelling indices of the EM cases in our study were similar to those reported by Meerang et al (25), they were significantly lower than those reported by Hmeljak et al (median, 67; mean, 63; range, 9.7-94.9; SD, 20.8) (26). This discrepancy in Survivin expression may be due to differences in staining technique, source of antibodies used for analysis, 

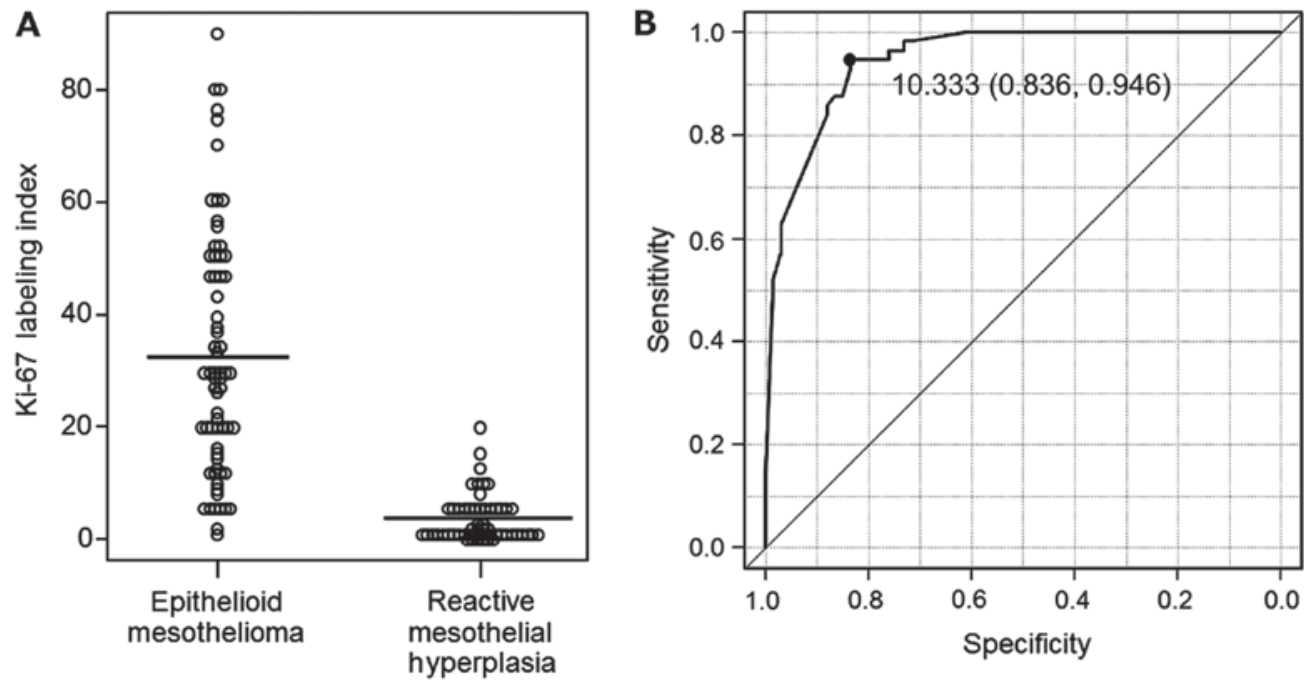

Figure 4. (A) Distributions of Ki-67 labelling index in epithelioid mesothelioma and reactive mesothelial hyperplasia. The horizontal line in the dot chart shows the mean. (B) ROC analysis. ROC curve was estimated using Ki-67 labelling index. Cut-off value based on the Youden index is also shown. ROC, receiver operating characteristic.

A

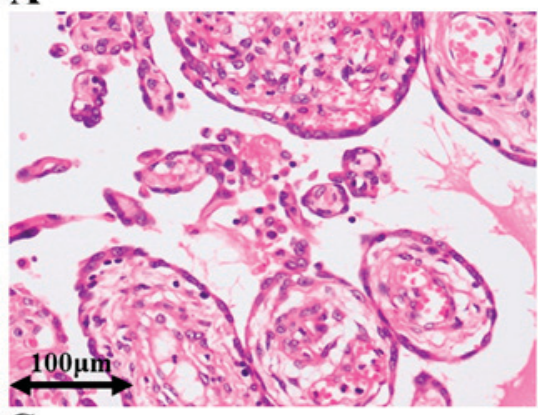

C

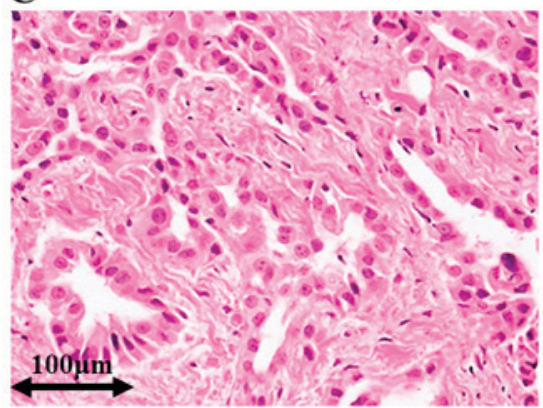

B

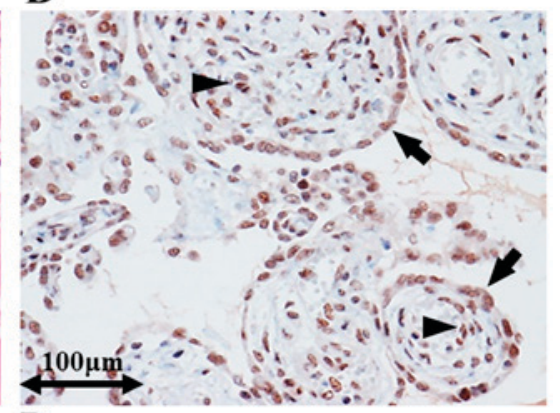

D

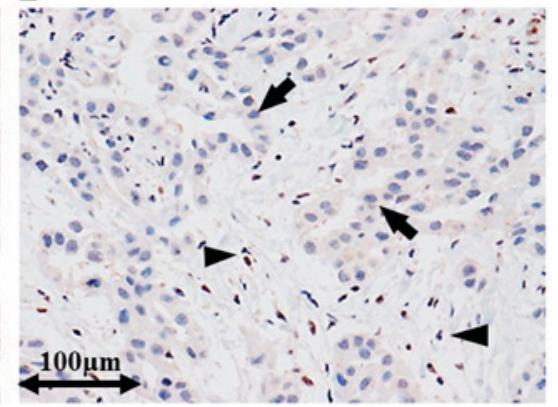

Figure 5. Representative histological images of BAP1 IHC. (A) RMH with H\&E stain. (B) BAP1 IHC in RMH. Nuclear staining of the mesothelial cells (arrows) demonstrated the same intensity as that of internal positive controls (arrowheads; stromal cells). (C) EM with H\&E stain. (D) BAP1 IHC in EM. Nuclear staining was not observed in tumour cells (loss of expression). Strong nuclear staining was observed in internal positive controls (arrowheads; stromal cells). IHC, immunohistochemistry; EM, epithelioid mesothelioma; RMH, reactive mesothelial hyperplasia; H\&E, haematoxylin and eosin; BAP1, BRCA1-associated protein 1.

and the quantification technique. In our study, we used fully automated immunohistochemical staining utilising equipment from Roche for reproducible results. We also used commercially available antibodies from reputable sources and evaluated nuclear reactivity alone. Evaluation of nuclear reactivity was reproducible and was independently confirmed by 3 pathologists.

Several studies have determined that germline mutations in the gene for $B A P l$ predispose individuals to developing various tumours, including MM, cutaneous melanocytic tumours, uveal melanoma, lung adenocarcinoma, and meningioma (31). These studies suggest that germline mutations in $B A P 1$ result in a 'tumour predisposition syndrome' linking BAP1 to many other cancers. Somatic mutations in the BAPl gene have also been relatively frequently reported in MMs, uveal melanomas, and renal cell carcinomas (31). BAP1 is encoded by the BAPI gene, which is located on the short arm of chromosome 3 (3p21). BAP1 is a deubiquitinase targeting histones and the host cell factor-1 transcriptional co-factor, and plays a role in transcriptional regulation, chromatin modulation, cell cycle 
Table II. Sensitivity, specificity, PPVs, NPVs and diagnostic accuracies of each marker and combinations of two markers for the differential diagnosis between epithelioid mesothelioma and reactive mesothelial hyperplasia.

\begin{tabular}{|c|c|c|c|c|c|}
\hline Immunohistochemical findings & Sensitivity (\%) & Specificity (\%) & PPV $(\%)$ & NPV $(\%)$ & Accuracy $(\%)$ \\
\hline Survivin-positive & 67.7 & 100.0 & 100.0 & 77.8 & 84.8 \\
\hline BAP1-loss & 66.2 & 100.0 & 100.0 & 75.7 & 83.6 \\
\hline Ki-67-positive & 85.1 & 87.5 & 89.1 & 83.1 & 86.2 \\
\hline Survivin-positive and/or BAP1-loss & 89.8 & 100.0 & 100.0 & 92 & 95.3 \\
\hline Both Survivin-positive and BAP1-loss & 39.0 & 100 & 100.0 & 65.7 & 71.9 \\
\hline Survivin-positive and/or Ki-67-positive & 91.1 & 86.3 & 87.9 & 89.8 & 88.8 \\
\hline Both Survivin-positive and Ki-67-positive & 66.1 & 100.0 & 100.0 & 72.9 & 82.2 \\
\hline BAP1-loss and/or Ki-67-positive & 96.9 & 92.1 & 94.3 & 95.9 & 94.8 \\
\hline Both BAP1-loss and Ki-67-positive & 53.8 & 100 & 100.0 & 64.3 & 74.8 \\
\hline
\end{tabular}

PPV, positive predictive values; NPV, negative predictive values; BAP1, BRCA1-associated protein 1.

regulation, and DNA repair $(31,32)$. Several different alterations in the $B A P l$ gene have been described, including large deletions of exons leading to loss of the $\mathrm{N}$-terminal region, or to premature protein termination, focal deletions, frameshift mutations due to insertions or deletions, splice site mutations, and base substitutions leading to nonsense and missense mutations. Frameshift mutations and missense and nonsense substitutions are the most common sequence alterations. Truncating mutations frequently result in loss of the nuclear localization signal and/or the C-terminal protein-binding domain, while missense mutations interfere with the ubiquitin hydrolase function of BAP1 (31). As the detection of these alterations in $B A P 1$ has been made possible in recent years using immunohistochemistry (IHC), immunohistochemical detection of BAP1 loss has also been reported to be useful in distinguishing MM from RMH. However, the sensitivity of this assay in differentiating MM from RMH does not exceed $70 \%$ (10-13). Several studies indicate that the loss of nuclear BAP1 expression as assessed by IHC is closely correlated with genetic alterations in BAP1 (33-35).

In the present study, the frequency of BAP1 loss in EM was $66.2 \%(49 / 74)$, similar to those found in previous reports (10-13). Recently, Hida et al reported a focal heterogeneous BAP1 staining pattern in mesothelioma cases (10). However, in our study, almost all EM cases had either a uniform positive staining pattern or completely negative staining for BAP1. There were some EM cases that appeared to have focal staining for BAP1; however, careful observation of these cases under high power magnification confirmed that these focal positive cells were in fact inflammatory cells infiltrating into the mesothelioma or stromal cells. We classified such cases as cases with no loss of BAP1 expression. This may be the reason for the observed heterogeneous BAP1 staining pattern in mesothelioma. However, other reasons, such as differences in staining techniques and improper processing of the tumour, may also contribute to apparent differences between studies.

The specificity of a Survivin labelling index of over $5 \%$ and a loss of BAP1 expression was $100 \%$. However, sensitivity of Survivin labelling index $(67.7 \%)$ and loss of BAP1 expression $(66.2 \%)$ alone are not sufficient for differential diagnosis. Although diagnostic accuracies of Survivin (84.8\%) and BAP1 $(83.6 \%)$ as single markers were inferior to that of EMA $(95.5 \%),(21)$ the diagnostic accuracy of the combination of Survivin and BAP1 (Survivin-positive and/or BAP1-loss) was $95.3 \%$, which was almost similar to EMA. Recently, Shinozaki-Ushiku et al proposed using a combination of BAP1 and enhancer of zeste homolog 2 (EZH2) expression to differentiate between MM from RMH; the sensitivity of this combination was $90 \%$, while the specificity was absolute (36). The sensitivity $(89.8 \%)$ and specificity $(100 \%)$ of the combination of Survivin and BAP1 IHC in this study was comparable to those of previous reports (36).

A positive correlation between nuclear Survivin and $\mathrm{Ki}-67$ labelling indices was previously reported by Meerang et al (25). We observed a similar correlation between Survivin and Ki-67 labelling indices in our study (data not shown). Although this correlation was present in both EM and RMH, it was more conspicuous in EM. Ki-67 protein is present during all active phases of the cell cycle (G1, S, G2, and mitosis), but is absent in resting cells (G0). Therefore, Ki-67 is well known as a so-called 'proliferation marker', and the Ki-67 labelling index is often correlated with the clinical course of cancer $(37,38)$. On the other hand, nuclear Survivin plays important roles in the regulation of mitosis. Survivin expression is found to be dominant only in the G2/M phase, and Survivin is known to localize to components of the mitotic spindle during the metaphase and anaphase of mitosis $(39,40)$. Therefore, both nuclear Survivin and Ki-67 may be considered proliferation markers. We can thus explain both the high expression of Survivin and Ki-67 in EM compared to RMH, and the positive correlation between the nuclear Survivin and Ki-67 labelling indices.

Although various studies have reported the usefulness of Ki-67 IHC in differentiating EM from RMH, (14-17) it is not routinely utilized for the confirmation of mesothelioma due to its low sensitivity and specificity.

The sensitivity, specificity, and diagnostic accuracy of Ki-67 $(85.1,87.5$, and $86.2 \%$, respectively) in this study were almost the same or slightly higher compared with previous reports $(14,15,17)$. These values were relatively high but not sufficient for differential diagnosis by single marker. However, the diagnostic accuracy 
of the combination of Ki-67 and BAP1 was 94.8\%, which was almost the same as that of the combination of Survivin and BAP1.

We evaluated the utility of Survivin, BAP1, and Ki-67 IHC in distinguishing EM from RMH. Based on our results, 'Survivin-positive and/or BAP1-loss' finding strongly suggest EM, therefore we recommend the use of a combination of Survivin and BAP1. In addition, further evaluation of the Ki-67 labelling index may be useful for accurate differential diagnosis.

\section{Acknowledgements}

The authors would like to thank the Technical Centre of Hiroshima University for technical assistance. This study was funded in part by the Japanese Ministry of Health, Labour, and Welfare.

\section{References}

1. Robinson BW, Musk AW and Lake RA: Malignant mesothelioma. Lancet 366: 397-408, 2005.

2. Roggli VL, Sharma A, Butnor KJ, Sporn T and Vollmer RT: Malignant mesothelioma and occupational exposure to asbestos: A clinicopathological correlation of 1445 cases. Ultrastruct Pathol 26: 55-65, 2002.

3. Delgermaa V, Takahashi K, Park EK, Le GV, Hara T and Sorahan T: Global mesothelioma deaths reported to the World Health Organization between 1994 and 2008. Bull World Health Organ 89: 716-724, 2011.

4. Murayama T, Takahashi K, Natori Y and Kurumatani N: Estimation of future mortality from pleural malignant mesothelioma in Japan based on an age-cohort model. Am J Ind Med 49: 1-7, 2006.

5. Churg A, Roggli V, Galateau-Salle F, Cagle PhT, Gibbs AR, Hasleton PhS, Henderson DW, Vignaud JM, Inai K, Praet M, et al: Tumours of the pleura. In: WHO Classification of Tumours of the Lung, Pleura, Thymus and Heart. Travis WD, Brambilla E, Burke AP, Marx A and Nicholson AG (eds). IARC Press, Lyon, pp153-181, 2015.

6. Husain AN, Colby T, Ordonez N, Krausz T, Attanoos R, Beasley MB, Borczuk AC, Butnor K, Cagle PT, Chirieac LR, et al: Guidelines for pathologic diagnosis of malignant mesothelioma: 2012 update of the consensus statement from the International Mesothelioma Interest Group. Arch Pathol Lab Med 137: 647-667, 2013

7. Ordóñez NG: Application of immunohistochemistry in the diagnosis of epithelioid mesothelioma: A review and update. Hum Pathol 44: 1-19, 2013.

8. Kushitani K, Amatya VJ, Okada Y, Katayama Y, Mawas AS, Miyata Y, Okada M, Inai K, Kishimoto T and Takeshima Y: Utility and pitfall of immunohistochemistry in the differential diagnosis between epithelioid mesothelioma and poorly differentiated lung squamous cell carcinoma. Histopathology 70: 375-384, 2017

9. Minato H, Kurose N, Fukushima M, Nojima T, Usuda K, Sagawa M, Sakuma T, Ooi A, Matsumoto I, Oda M, et al: Comparative immunohistochemical analysis of IMP3, GLUT1, EMA, CD146, and desmin for distinguishing malignant mesothelioma from reactive mesothelial cells. Am J Clin Pathol 141: 85-93, 2014.

10. Hida T, Hamasaki M, Matsumoto S, Sato A, Tsujimura T, Kawahara K, Iwasaki A, Okamoto T, Oda Y, Honda $\mathrm{H}$ and Nabeshima K: BAP1 immunohistochemistry and p16 FISH results in combination provide higher confidence in malignant pleural mesothelioma diagnosis: ROC analysis of the two tests. Pathol Int 66: 563-570, 2016.

11. Hwang HC, Sheffield BS, Rodriguez S, Thompson K, Tse $\mathrm{CH}$, Gown AM and Churg A: Utility of BAP1 immunohistochemistry and p16 (CDKN2A) FISH in the diagnosis of malignant mesothelioma in effusion cytology specimens. Am J Surg Pathol 40: 120-126, 2016

12. McGregor SM, Dunning R, Hyjek E, Vigneswaran W, Husain AN and Krausz T: BAP1 facilitates diagnostic objectivity, classification, and prognostication in malignant pleural mesothelioma. Hum Pathol 46: 1670-1678, 2015.
13. Cigognetti M, Lonardi S, Fisogni S, Balzarini P, Pellegrini V, Tironi A, Bercich L, Bugatti M, Rossi G, Murer B, et al: BAP1 (BRCA1-associated protein 1) is a highly specific marker for differentiating mesothelioma from reactive mesothelial proliferations. Mod Pathol 28: 1043-1057, 2015.

14. Kimura F, Okayasu I, Kakinuma H, Satoh Y, Kuwao S, Saegusa M and Watanabe J: Differential diagnosis of reactive mesothelial cells and malignant mesothelioma cells using the cell proliferation markers minichromosome maintenance protein 7, geminin, topoisomerase II alpha and Ki-67. Acta Cytol 57: 384-390, 2013.

15. Kimura F, Kawamura J, Watanabe J, Kamoshida S, Kawai K, Okayasu I and Kuwao S: Significance of cell proliferation markers (Minichromosome maintenance protein 7, topoisomerase IIalpha and Ki-67) in cavital fluid cytology: Can we differentiate reactive mesothelial cells from malignant cells? Diagn Cytopathol 38: 161-167, 2010.

16. Hasteh F, Lin GY, Weidner N and Michael CW: The use of immunohistochemistry to distinguish reactive mesothelial cells from malignant mesothelioma in cytologic effusions. Cancer Cytopathol 118: 90-96, 2010

17. Taheri ZM, Mehrafza M, Mohammadi F, Khoddami M, Bahadori M and Masjedi MR: The diagnostic value of Ki-67 and repp86 in distinguishing between benign and malignant mesothelial proliferations. Arch Pathol Lab Med 132: 694-697, 2008.

18. Churg A, Sheffield BS and Galateau-Salle F: New markers for separating benign from malignant mesothelial proliferations: Are we there yet? Arch Pathol Lab Med 140: 318-321, 2016.

19. Hiroshima K, Wu D, Hasegawa M, Koh E, Sekine Y, Ozaki D, Yusa T, Walts AE, Marchevsky AM, Nabeshima K, et al: Cytologic differential diagnosis of malignant mesothelioma and reactive mesothelial cells with fish analysis of p16. Diagn Cytopathol 44: 591-598, 2016.

20. Walts AE, Hiroshima K, McGregor SM, Wu D, Husain AN and Marchevsky AM: BAP1 immunostain and CDKN2A (p16) FISH analysis: Clinical applicability for the diagnosis of malignant mesothelioma in effusions. Diagn Cytopathol 44: 599-606, 2016.

21. Kushitani K, Amatya VJ, Mawas AS, Miyata Y, Okada M and Takeshima Y: Use of anti-noxa antibody for differential diagnosis between epithelioid mesothelioma and reactive mesothelial hyperplasia. Pathobiology 83: 33-40, 2016.

22. Travis WD, Brambilla E, Nicholson AG, Yatabe Y, Austin JHM, Beasley MB, Chirieac LR, Dacic S, Duhig E, Flieder DB, et al: The 2015 World Health Organization Classification of Lung Tumors: Impact of genetic, clinical and radiologic advances since the 2004 classification. J Thorac Oncol 10: 1243-1260, 2015.

23. Kanda Y: Investigation of the freely available easy-to-use software 'EZR' for medical statistics. Bone Marrow Transplant 48: 452-458, 2013.

24. Garg H, Suri P, Gupta JC, Talwar GP and Dubey S: Survivin: A unique target for tumor therapy. Cancer Cell Int 16: 49, 2016.

25. Meerang M, Bérard K, Friess M, Bitanihirwe BK, Soltermann A, Vrugt B, Felley-Bosco E, Bueno R, Richards WG, Seifert B, et al: Low merlin expression and high survivin labeling index are indicators for poor prognosis in patients with malignant pleural mesothelioma. Mol Oncol 10: 1255-1265, 2016.

26. Hmeljak J, Erčulj N, Dolžan V, Pižem J, Kern I, Kovač V, Cemažar $\mathrm{M}$ and Cör A: Is survivin expression prognostic or predictive in malignant pleural mesothelioma? Virchows Arch 462: 315-321, 2013.

27. Chen S, Wang Y, An L, Fei ZT and Li T: The diagnostic value of survivin in malignant pleural effusion: A meta-analysis. Clin Chim Acta 441: 142-147, 2015.

28. Bertino P, Panigada M, Soprana E, Bianchi V, Bertilaccio S, Sanvito F, Rose AH, Yang H, Gaudino G, Hoffmann PR, et al: Fowlpox-based survivin vaccination for malignant mesothelioma therapy. Int J Cancer 133: 612-623, 2013.

29. De Cesare M, Cominetti D, Doldi V, Lopergolo A, Deraco M, Gandellini P, Friedlander S, Landesman Y, Kauffman MG, Shacham S, et al: Anti-tumor activity of selective inhibitors of XPO1/CRM1-mediated nuclear export in diffuse malignant peritoneal mesothelioma: The role of survivin. Oncotarget 6: 13119-13132, 2015

30. Goričar K, Kovač V, Franko A, Dodič-Fikfak M and Dolžan V: Serum survivin levels and outcome of chemotherapy in patients with malignant mesothelioma. Dis Markers 2015: 316739, 2015.

31. Murali R, Wiesner T and Scolyer RA: Tumours associated with BAP1 mutations. Pathology 45: 116-126, 2013.

32. Scheuermann JC, de Ayala Alonso AG, Oktaba K, Ly-Hartig N, McGinty RK, Fraterman S, Wilm M, Muir TW and Müller J: Histone H2A deubiquitinase activity of the Polycomb repressive complex PR-DUB. Nature 465: 243-247, 2010. 
33. Bott M, Brevet M, Taylor BS, Shimizu S, Ito T, Wang L, Creaney J, Lake RA, Zakowski MF, Reva B, et al: The nuclear deubiquitinase BAP1 is commonly inactivated by somatic mutations and 3p21.1 losses in malignant pleural mesothelioma. Nat Genet 43: 668-672, 2011

34. Testa JR, Cheung M, Pei J, Below JE, Tan Y, Sementino E, Cox NJ, Dogan AU, Pass HI, Trusa S, et al: Germline BAP1 mutations predispose to malignant mesothelioma. Nat Genet 43: 1022-1025, 2011.

35. Yoshikawa Y,Sato A,TsujimuraT,EmiM,Morinaga T,FukuokaK, Yamada S, Murakami A, Kondo N, Matsumoto S, et al: Frequent inactivation of the BAP1 gene in epithelioid-type malignant mesothelioma. Cancer Sci 103: 868-874, 2012.

36. Shinozaki-Ushiku A, Ushiku T, Morita S, Anraku M, Nakajima J and Fukayama M: Diagnostic utility of BAP1 and EZH2 expression in malignant mesothelioma. Histopathology 70: 722-733, 2017.

37. Scholzen T and Gerdes J: The Ki-67 protein: From the known and the unknown. J Cell Physiol 182: 311-322, 2000.
38. Brown DC and Gatter KC: Monoclonal antibody Ki-67: Its use in histopathology. Histopathology 17: 489-503, 1990.

39. Altieri DC: Validating survivin as a cancer therapeutic target. Nat Rev Cancer 3: 46-54, 2003.

40. Kim JY, Chung JY, Lee SG, Kim YJ, Park JE, Yoo KS, Yoo YH, Park YC, Kim BG and Kim JM: Nuclear interaction of Smac/DIABLO with survivin at $\mathrm{G} 2 / \mathrm{M}$ arrest prompts docetaxel-induced apoptosis in DU145 prostate cancer cells. Biochem Biophys Res Commun 350: 949-954, 2006.

(i)(3) This work is licensed under a Creative Commons Attribution-NonCommercial-NoDerivatives 4.0 International (CC BY-NC-ND 4.0) License. 